J. Gastroenterol. Hepatol. Erkr. 2018 · 16:56-62 https://doi.org/10.1007/s41971-018-0028-8

Online publiziert: 24. Mai 2018

(c) Der/die Autor(en) 2018

CrossMark

\section{Gernot Zollner ${ }^{1} \cdot$ Jürgen Prattes ${ }^{2} \cdot$ Alexandra Nemeth}

' Klinische Abteilung für Gastroenterologie und Hepatologie, Universitätsklinik für Innere Medizin, Medizinische Universität Graz, Graz, Österreich

${ }^{2}$ Sektion für Infektiologie und Tropenmedizin, Universitätsklinik für Innere Medizin, Medizinische Universität Graz, Graz, Österreich

\title{
Primär biliäre Cholangitis in Graz: Präsentation, Verlauf und Outcome
}

\section{Einleitung}

Die primär biliäre Cholangitis ( $\mathrm{PBC})$, früher als primär biliäre Zirrhose bekannt, ist eine autoimmune Erkrankung, die vor allem Frauen zwischen dem 30. und 65. Lebensjahr betrifft [1]. Die Umbenennung der Erkrankung von Zirrhose zu Cholangitis im Jahr 2015 ist sehr zu begrüßen, da nur ein Teil der PBCPatienten eine Zirrhose entwickelt und somit die Stigmatisierung und auch Beunruhigung der Patienten durch die unglückliche Namensgebung nun wegfällt [2].

Die meisten Daten zur Präsentation, zum Verlauf und auch zur Prognose der PBC stammen aus Nordamerika, Großbritannien, Island, Spanien und Australien. Da diese Erkrankung offensichtlich in den verschiedensten Teilen der Welt unterschiedlich schwer verläuft, ist es unklar, ob die Datenlage aus den oben erwähnten Regionen auch auf unser Patientenkollektiv angewandt werden kann. Daten zum natürlichen Verlauf der Erkrankung sind unerlässlich, um die Prognose der Patienten besser abschätzen zu können und sie in dieser Hinsicht auch ausreichend beraten zu können. Ziel dieser Arbeit war es daher, demographische und klinische Daten, den Verlauf und das Outcome von PBC-Patienten an der Klinischen Abteilung für Gastroenterologie und Hepatologie der Medizinischen Universität Graz retrospektiv zu erheben.

\section{Methoden}

Die Identifikation aller Patienten mit der Diagnose PBC erfolgte nach einer Stichwortabfrage über das Krankenhausdokumentationssystem openMEDOCs. In die Arbeit eingeschlossen wurden alle $\mathrm{Pa}$ tienten mit der Diagnose PBC, die im Zeitraum zwischen dem 01.01.2004 und dem 30.09.2015 an der Leberambulanz der Klinischen Abteilung für Gastroenterologie und Hepatologie in Betreuung waren. Die Diagnose PBC wurde retrospektiv nochmals geprüft. Nur Patienten mit einer Erhöhung der alkalischen Phosphatase (AP) länger als 6 Monate und erhöhten antimitochondrialen Antikörpern (AMA; >1:40) bzw. mit einer mit PBC zu vereinbaren Leberhistologie entsprechend den bei Studienbeginn gültigen Diagnosekriterien der European Association for the Study of the Liver (EASL; [3]) wurden eingeschlossen.

Die Krankenakten wurden zum Zeitpunkt der Erstdiagnose und der letzten Visite an der Ambulanz (Follow-up) auf folgende Zielgrößen retrospektiv hin untersucht: Zeitpunkt der Erstdiagnose und der letzten Visite, Krankheitsdauer, Geschlecht, Körpergewicht, Laborbiochemie (Aspartat-Aminotransferase [AST], Alanin-Amino-Transferase [ALT], AP, Bilirubin, Thrombozyten, Gallensäuren, AMA), klinische Symptome (Pruritus, Müdigkeit, Oberbauchschmerzen, Sicca-Symptomatik, Ikterus, hepatische Enzephalopathie), Leberhistologie, Bildgebung, FibroScan ${ }^{\circledR}$-Messungen (Echosens, Paris, Frankreich), Dosis der Ur- sodesoxycholsäuretherapie, Ansprechen auf die Therapie mit Ursodesoxycholsäure, Auftreten einer Leberzirrhose, eines hepatozellulären Karzinoms, Tod oder Lebertransplantation. Alle Daten werden als Median mit Spannweite (Minimum - Maximum) angegeben.

\section{Ergebnisse}

Insgesamt wurden 156 Patienten mit der Diagnose PBC identifiziert, die an unserer Abteilung zwischen den Jahren 2004 und 2015 in Betreuung waren (siehe - Abb. 1). 95 Patienten erfüllten retrospektiv die Diagnosekriterien der PBC und wurden in die finalen Analysen aufgenommen. 38 Patienten mussten aus folgenden Gründen aus den Analysen ausgeschlossen werden: (a) Bei 30 Patienten waren Labordaten zum Zeitpunkt der Erstdiagnose (die bei diesen Patienten in den 80er- und 90er-Jahren des letzten Jahrhunderts lag) nicht mehr archiviert, sodass die Diagnosekriterien der EASL [3] auf diese Fälle nicht anwendbar waren; (b) bei 8 Fällen wurden die Diagnosekriterien nicht erfüllt. Bei 28 Patienten wurde die Diagnose vor dem 01.01.2004 gestellt, bei 67 Fällen zwischen Jänner 2004 und September 2015.

Weitere 23 Patienten hatten zum Zeitpunkt des Erstkontakts in unserer Ambulanz zwar positive AMA, aber keine erhöhte AP und erfüllten somit nicht die Kriterien einer PBC. Diese Patienten wurden in unserer Ambulanz weiter betreut und als "prächolestatische PBC“ 


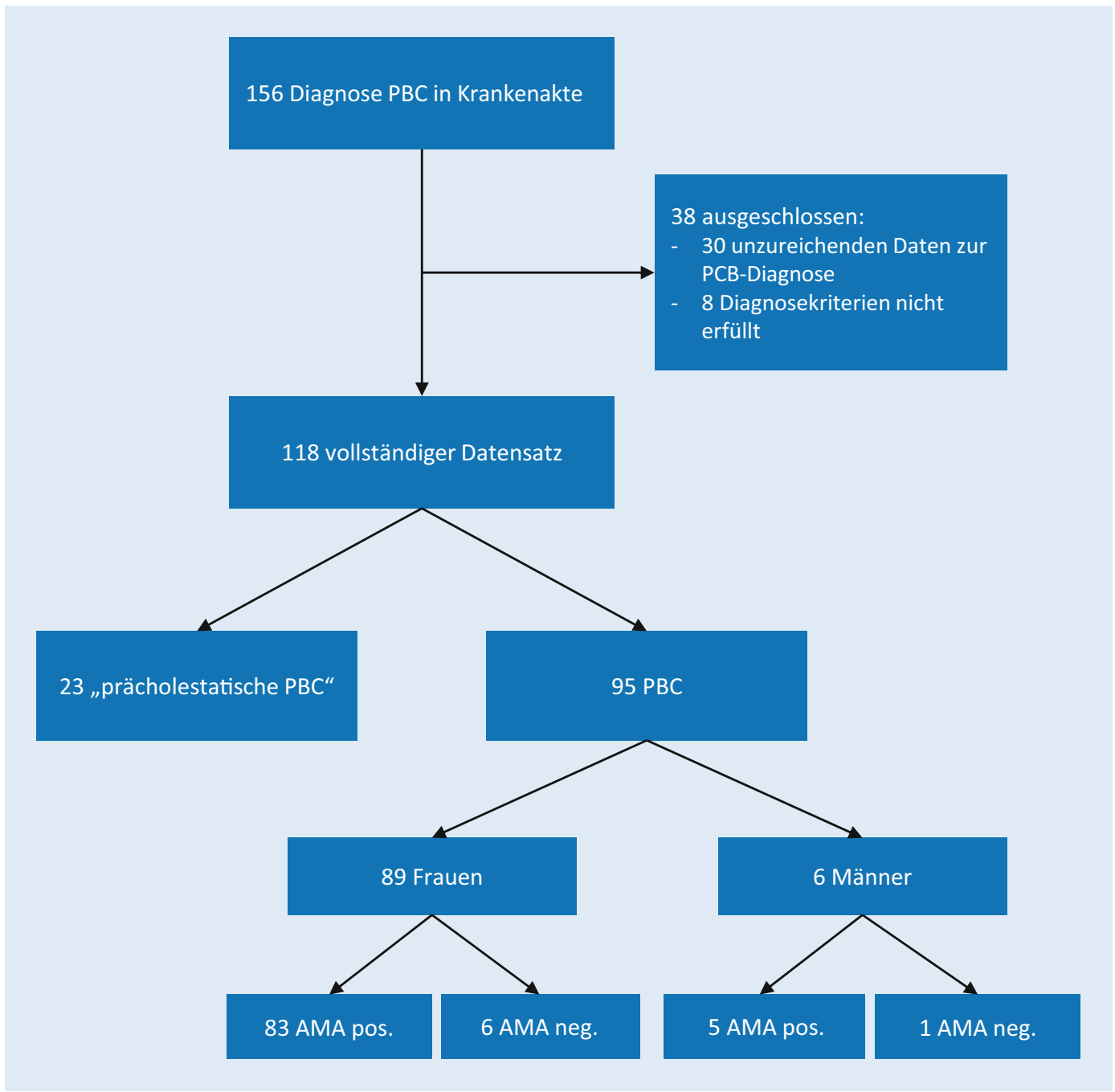

Abb. $1<$ Flowchart zur Patientenidentifikation. $A M A$ antimitochondriale Antikörper, $P B C$ primär biliäre Cholangitis

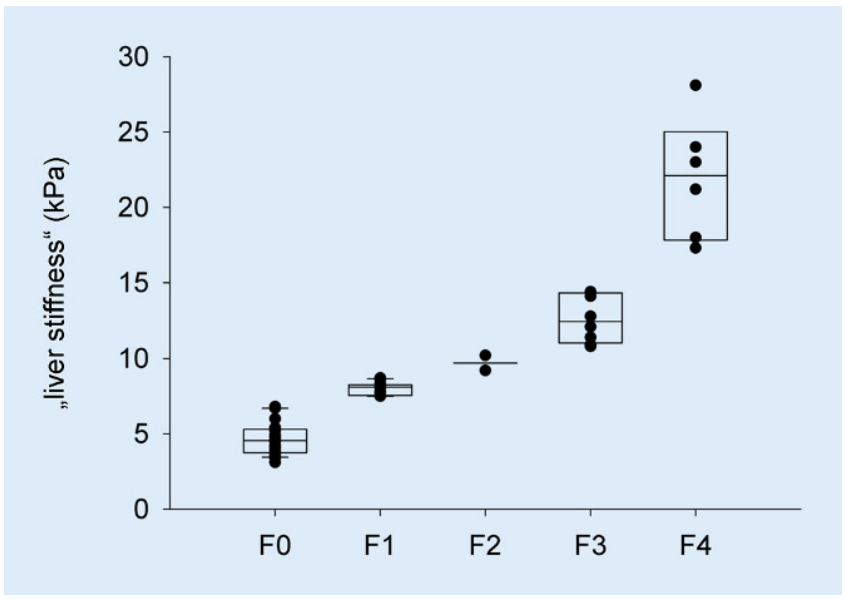

Abb. $2<$ Transiente Leberelastograpie mittels FibroScan ${ }^{\circledR}$ zur Messung der Lebersteifigkeit (,/liver stiffness"). F Fibrose in dieser Arbeit getrennt von den $95 \mathrm{~Pa}$ tienten mit PBC ausgewertet (• Abb. 1). Die Mehrzahl der Patienten waren Frauen $(n=89)$. Lediglich 6 Männer fanden sich in unserer Kohorte (• Abb. 1). Das mediane Alter bei Erstdiagnose betrug
53 Jahre (26-84 Jahre) und unterschied sich nicht signifikant zwischen Frauen (51 Jahre) und Männern (57 Jahre). Sieben Fälle wurden vor dem 40. Lebensjahr diagnostiziert. Positive AMA lagen bei
87 Patienten (92\%) vor, 8 (8\%) hatten eine AMA-negative PBC.

\section{Stadium der Lebererkrankung und Symptome zum Zeitpunkt der Erstdiagnose}

Eine Leberhistologie wurde bei $35 \mathrm{~Pa}$ tienten bei der Erstdiagnose gewonnen. Die Mehrzahl hatte eine PBC im Stadium I ( $n=28)$ nach Ludwig [4], 5 Patienten eine PBC im Stadium II und jeweils eine Patientin im Stadium III und IV. Bei einer weiteren Patientin wurde bei der Erstdiagnose durch bildgebende Verfahren das Vorliegen einer Leberzirrhose diagnostiziert. Insgesamt hatten $2 \mathrm{~Pa}$ tientinnen bei der Erstdiagnose bereits eine Leberzirrhose. Eine Komplikation der Zirrhose oder klinische Hinweise auf eine fortgeschrittene Erkrankung, wie Ikterus, Aszites, hepatozelluläres Karzinom 
oder Varizenblutung, fanden sich bei der Erstdiagnose nicht. Bei 6 Patienten bestand bei der Erstdiagnose ein OverlapSyndrom zu einer Autoimmunhepatitis. Einer dieser Fälle war mit einer Leberzirrhose assoziiert.

Bei der Erstdiagnose waren $37 \mathrm{~Pa}$ tienten (39\%) symptomatisch. Häufigstes Symptom war Pruritus bei 17 Patienten, gefolgt von Oberbauchschmerzen $(n=14)$, Sicca-Symptomatik $(n=13)$ und Müdigkeit $(n=11)$.

\section{Stadium der Lebererkrankung und Komplikationen im Follow-up}

Die mediane Beobachtungsdauer der Kohorte vom Zeitpunkt der Erstdiagnose bis zur letzten Visite (Follow-up) an unserer Abteilung betrug 7 Jahre (1-21 Jahre). Messungen der Lebersteifigkeit mittels FibroScan ${ }^{\circledR}$ erfolgten bei 52 Patienten im Krankheitsverlauf. Die Mehrzahl der Patienten hatte eine F0/F1-Fibrose $(n=36,69 \%), 2$ (4\%) eine F2-Fibrose und 14 Patienten $(27 \%)$ eine höhergradige F3- $(n=8)$ oder F4-Fibrose $(n=6$; - Abb. 2). Als Cut-off-Werte für die Fibrosegrade wurden die für die $\mathrm{PBC}$ von Corpechot et al. im Jahr 2012 ermittelten Werte herangezogen [5]. Über dem Cut-off-Wert von $9,6 \mathrm{kPa}$, der mit einem 5-fach erhöhten Risiko für eine Dekompensation der Lebererkrankung, Lebertransplantation oder Tod assoziiert ist [6], lagen 15 Patienten. Zum Zeitpunkt der Follow-up-Untersuchung hatten zusätzlich zu den 6 Patientinnen mit einer im FibroScan ${ }^{\circledR}$ ermittelten F4-Fibrose 4 weitere Fälle die radiologische Diagnose einer Leberzirrhose erhalten. Somit lagen in unserer PBC-Kohorte 10 Fälle (11\%) mit Leberzirrhose im Follow-up vor. Bei 2 Patienten war die Zirrhose bereits bei der Erstdiagnose nachgewiesen, 8 Patienten hatten sie erst im Verlauf entwickelt. Die Mehrzahl der Fälle wies ein ChildStadium A $(n=8)$ auf, jeweils eine Patientin hatte eine Child-B- oder -C-Zirrhose. Zwei Patientinnen entwickelten Ösophagusvarizen, 2 einen Ikterus, eine ein hepatozelluläres Karzinom. Aszites fand sich bei einer Patientin. Varizenblutungen oder hepatische Enzephalopathien konnten in unserer Kohorte nicht beobachtet werden. Zwei Todesfälle wurden

J. Gastroenterol. Hepatol. Erkr. 2018 · 16:56-62 https://doi.org/10.1007/s41971-018-0028-8 (c) Der/die Autor(en) 2018

\section{G. Zollner $\cdot$ J. Prattes $\cdot$ A. Nemeth}

\section{Primär biliäre Cholangitis in Graz: Präsentation, Verlauf und Outcome}

\section{Zusammenfassung}

Präsentation, klinischer Verlauf und Outcome von Patienten mit primär biliärer Cholangitis (PBC) sind in Mitteleuropa nicht ausreichend beschrieben. Diese Arbeit untersucht demographische und klinische Daten und charakterisiert den Krankheitsverlauf der PBC am Patientenkollektiv am Universitätsklinikum Graz. Die Krankenakten von Patienten mit der Diagnose PBC, die im Zeitraum von 2004-2015 am Universitätsklinikum Graz in Betreuung waren, wurden retrospektiv untersucht. Erhoben wurden die Daten zum Zeitpunkt der Erstdiagnose und bei der letzten Kontrolle (Follow-up). Insgesamt wurden 95 Patienten (89 Frauen 6 Männer) identifiziert. Das mediane Alter be Erstdiagnose betrug 53 Jahre. Die alkalische Phosphatase (AP) war bei Erstdiagnose bei $60 \%$ der Patienten um weniger als das 2-Fache der Norm erhöht. 39\% der Patienten wiesen bei Erstdiagnose Symptome, wie Pruritus, Müdigkeit, Oberbauchbeschwerden oder eine Sicca-Symptomatik, auf. Eine Leberhistologie bei der Erstdiagnose lag bei $35 \%$ aller Fällen vor (histologisches Stadium I nach Ludwig, $n=28$; Stadium II, $n=5$; Stadium III, $n=1$; Stadium IV, $n=1$ ). Das mediane Follow-up betrug 7 (1-21) Jahre. Die Mehrzahl der Patienten (69\%) hatte in der FibroScan ${ }^{\circledR}$-Messung im Followup eine F0/F1-Fibrose. Eine Leberzirrhose lag in 10 Fällen (11\%) vor. Zwei mit der PBC assoziierte Todesfälle wurden verzeichnet. Ein inadäquates Ansprechen auf die Therapie mit Ursodesoxycholsäure lag in $30 \%$ aller Fälle vor. Zusammenfassend sind in unserem Kollektiv eine deutliche Erhöhung der AP ( $>2$ bis 4-Fache der Norm) und ein schlechtes Outcome (Entwicklung einer Zirrhose, Tod, Transplantation) seltener als in der Literatur angegeben, während demographische Daten durchaus mit denen aus anderen Arbeiten vergleichbar sind.

\section{Schlüsselwörter}

Cholestase - Alkalische Phosphatase . Ursodesoxycholsäure · FibroScan ${ }^{\circledR}$

\section{Primary Biliary Cholangitis in Graz: Presentation, Course, and Outcome}

\section{Abstract}

The presentation, clinical course, and outcome in patients with primary biliary cholangitis (PBC) may vary in different geographic regions. Data on this disease entity in central Europe are scarce. Therefore, the aim of this work was to characterize PBC patients at the Medical University of Graz. Data at time of diagnosis and from the last visit (follow-up) were obtained retrospectively from records of patients at our institution from 2004-2015. We identified 95 patients with PBC (89 women, 6 men). Median age at time of diagnosis was 53 years. Alkaline phosphatase (AP) was elevated less than 2-fold above the upper limit of norm in $60 \%$. Clinical symptoms (pruritus, fatigue, upper quadrant abdominal pain, keratoconjunctivitis sicca) were present in $39 \%$ of cases. Liver histology was available in
$35 \%$ (stage I, $n=28$; stage II, $n=5$; stage III, $n=1$; stage IV, $n=1$ ). Using FibroScan ${ }^{\circledR}$, $69 \%$ of patients had F0 or F1 liver fibrosis. Liver cirrhosis at follow-up was present in 10 patients (11\%). Two liver-related deaths were encountered. Inadequate response to therapy with ursodeoxycholic acid was found in $30 \%$ of cases. In summary, patient outcome in our population seems to be markedly better than reported before, while the demographic data are comparable with other published studies. This might be attributed to lower levels of AP at the time of diagnosis compared to other studies.

\section{Keywords}

Cholestasis - Alkaline phosphatase . Ursodeoxycholic acid · FibroScan ${ }^{\circledR}$ 


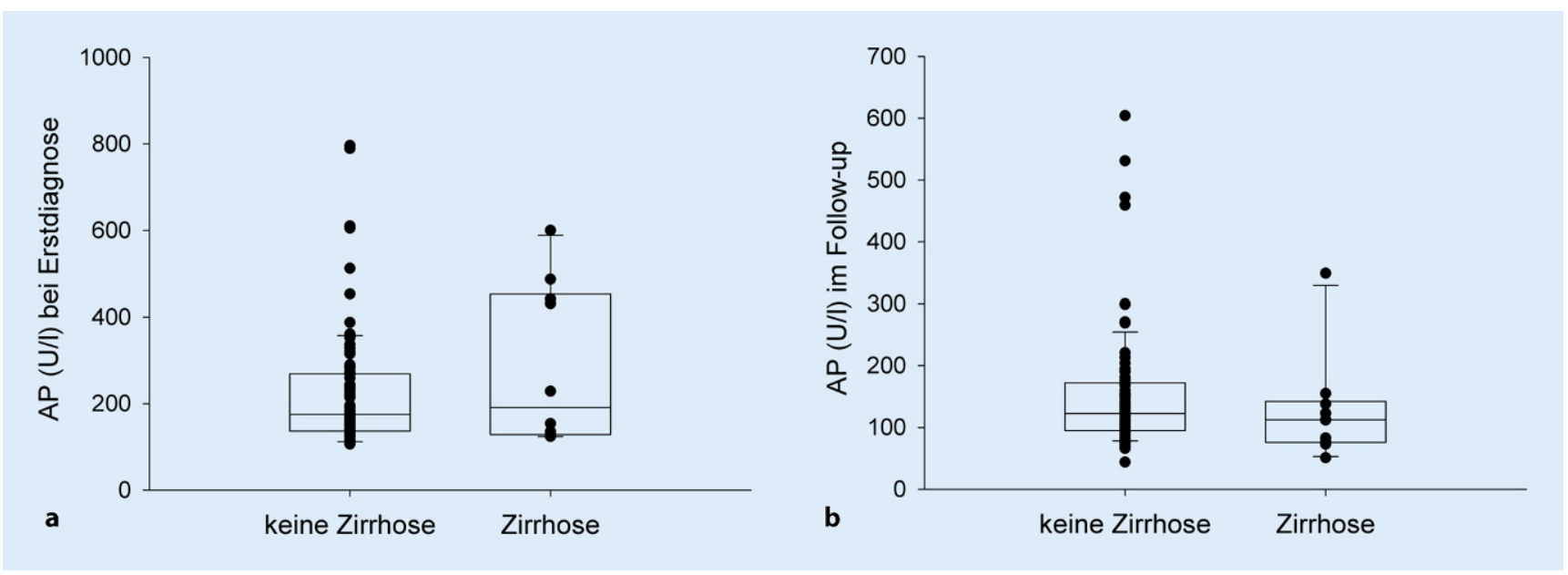

Abb. 3 \ Höhe der alkalischen Phosphatase (AP) bei derErstdiagnose (a) und im Follow-up (b) in Abhängigkeit des Vorliegens einer Leberzirrhose

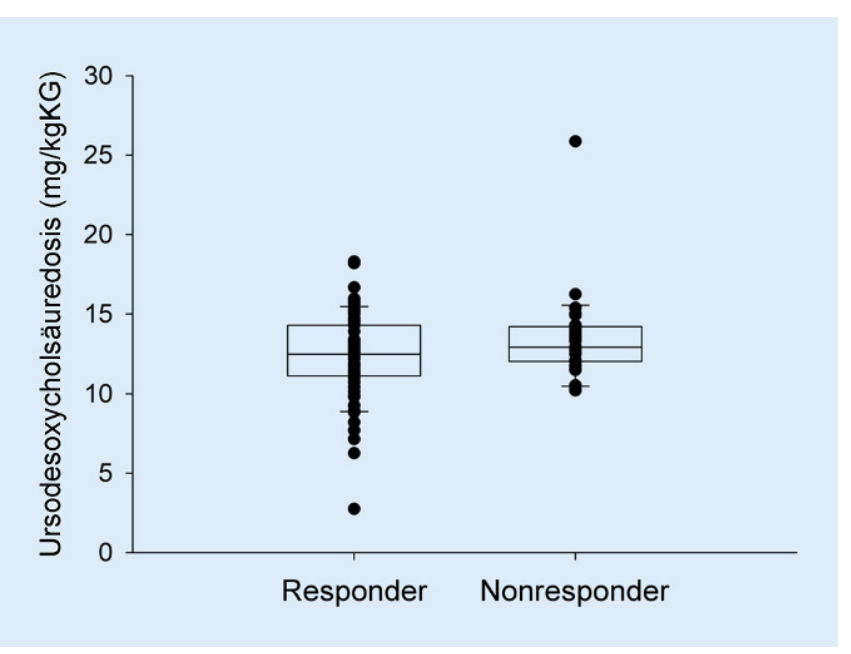

Abb. $4<$ Ursodesoxycholsäuredosis in Abhängigkeit des Ansprechens auf die Therapie beobachtet: Eine Patientin verstarb an einem hepatozellulären Karzinom, eine weitere durch Leberversagen. Bei keinem Patient wurde eine Lebertransplantation durchgeführt.

\section{Laborbiochemie bei Erstdiagnose und Follow-up}

Die AP war bei der Erstdiagnose bei den meisten Fällen nur moderat erhöht (Median 175 U/1, 106-796 U/l), im Follow-up lag sie im Median bei $121 \mathrm{U} / \mathrm{l}$ (44-604 U/l). Die AP und weitere Laborwerte bei der Erstdiagnose und im Follow-up sind in $\bullet$ Tab. 1 angeführt. Die absoluten Werte der AP unterschieden sich weder bei der Erstdiagnose ( $\bullet$ Abb. $\mathbf{3 a}$ ) noch im Follow-up (• Abb. 3b) zwischen Patienten mit oder ohne Leberzirrhose zum Zeitpunkt der Follow-up-Untersu- chung. Der Großteil der Fälle hatte bei der Erstdiagnose AP-Werte zwischen dem 1- und 2-Fachen des oberen Grenzwerts (- Tab. 2). In der Gruppe der Patienten mit maximal 2-fach erhöhter BaselineAP entwickelten nur 9\% eine Leberzirrhose im Verlauf, während $40 \%$ der Patienten mit einer mehr als 4-fach erhöhten Baseline-AP eine Leberzirrhose im Verlauf entwickelten (•Tab. 2). Im Gegensatz zu den Ergebnissen bei der Erstdiagnose hatte kein Patient mit einer AP über dem 4-Fachen der Norm im Followup eine Leberzirrhose (• Tab. 3 ).

\section{Therapie mit Ursodesoxycholsäure und Ansprechen auf die Therapie}

Alle Patienten wurden mit Ursodesoxycholsäure behandelt. Die mediane Dosis lag bei 13 mg/kg Körpergewicht (kg KG;
3-18) täglich. Die Ursodesoxycholsäuredosis war bei 39 Patienten (43\%) geringer als die empfohlene tägliche Dosis von $13-15 \mathrm{mg} / \mathrm{kg} \mathrm{KG}$. Das Ansprechen auf die Therapie entsprechend definierten Kriterien (wie Paris-I/II-, Toronto-, Barcelona-Kriterien), die den Laborverlauf nach 6-24 Monaten mit einbeziehen, kann aus unseren Daten nicht direkt abgelesen werden, da die Labordaten zu den entsprechenden Zeitpunkten nicht erhoben wurden. Die Ansprechraten auf Ursodesoxycholsäure werden in unserer Arbeit in Anlehnung an die Paris-II-Kriterien definiert: AP oder AST $\geq 1,5$-Fache des oberen Grenzwerts des Normbereichs oder Bilirubin $\geq 1 \mathrm{mg} / \mathrm{dl}$ zum Zeitpunkt des Follow-ups. Anhand dieser Kriterien konnten 29 Patienten (30\%) als Nonresponder mit einem inadäquaten Ansprechen auf Ursodesoxycholsäure identifiziert werden. Das Auftreten einer Leberzirrhose wurde nur bei einem Nonresponder beobachtet. Eine Normalisierung der AP auf Ursodesoxycholsäure fehlte in insgesamt 58 Fällen (61\%). Es fand sich kein Unterschied in der mittleren Ursodesoxycholsäuredosis zwischen Respondern und Nonrespondern auf die Therapie (•Abb.4). Die Fälle mit unzureichender Dosis waren in $33 \%$ Nonresponder, die mit adäquater Dosis in $28 \%(p>0,05)$. Vier von 39 Fällen $(10 \%)$ mit zu niedriger Ursodesoxycholsäuredosis entwickelten eine Zirrhose. Unter adäquater Therapie waren dies 6 von 52 $(12 \% ; p>0,05)$. 


\begin{tabular}{|lll|}
\hline Tab. 1 & Laborbiochemie bei der Erstdiagnose und im Follow-up \\
& $\begin{array}{l}\text { Erstdiagnose } \\
\text { Median (Spannweite) }\end{array}$ & $\begin{array}{l}\text { Follow-up } \\
\text { Median (Spannweite) }\end{array}$ \\
\hline AP (U/l) & $175(106-796)$ & $121(44-604)$ \\
\hline Bilirubin $(\mathrm{mg} / \mathrm{dl})$ & $0,5(0,2-4,5)$ & $0,5(0,2-4,4)$ \\
ALT $(\mathrm{U} / \mathrm{l})$ & $52(15-383)$ & $28(10-136)$ \\
\hline AST $(\mathrm{U} / \mathrm{l})$ & $40(14-442)$ & $29(15-102)$ \\
\hline Thrombozyten $(\mathrm{G} / \mathrm{l})$ & $263(106-439)$ & $241(90-404)$ \\
\hline Gallensäuren $(\mu \mathrm{M})$ & $6,6(0,2-25)$ & $13,8(0,4-57)$ \\
\hline AST Aspartat-Aminotransferase, & ALT Alanin-Amino-Transferase, $A P$ alkalische Phosphatase \\
\hline
\end{tabular}

Tab. 2 Alkalische Phosphatase (AP) bei Erstdiagnose und Risiko für Leberzirrhose

\begin{tabular}{lll} 
& Anzahl der Patienten & $\begin{array}{l}\text { Anzahl der Patienten mit } \\
\text { Zirrhose im Follow-up }\end{array}$ \\
\hline $\mathrm{AP}>1 \times \mathrm{ULN} \leq 2 \times \mathrm{ULN}$ & $57(60 \%)$ & $5(9 \%)$ \\
$\mathrm{AP}>2 \times \mathrm{ULN} \leq 4 \times \mathrm{ULN}$ & $28(29 \%)$ & $1(4 \%)$ \\
$\mathrm{AP}>4 \times \mathrm{ULN}$ & $10(11 \%)$ & $4(40 \%)$
\end{tabular}

AST Aspartat-Aminotransferase, ALT Alanin-Amino-Transferase, AP alkalische Phosphatase, ULN "upper limit of norm", oberer Grenzwert des Normbereichs

\begin{tabular}{|c|c|c|}
\hline & Anzahl der Patienten & $\begin{array}{l}\text { Anzahl der Patienten mit } \\
\text { Zirrhose im Follow-up }\end{array}$ \\
\hline$A P \leq 1 \times U L N$ & 35 (38\%) & $4(11 \%)$ \\
\hline $\mathrm{AP}>1 \times \mathrm{ULN} \leq 2 \times \mathrm{ULN}$ & $46(49 \%)$ & $5(11 \%)$ \\
\hline $\mathrm{AP}>2 \times \mathrm{ULN} \leq 4 \times \mathrm{ULN}$ & $6(6 \%)$ & $1(17 \%)$ \\
\hline $\mathrm{AP}>4 \times \mathrm{ULN}$ & $6(6 \%)$ & 0 \\
\hline
\end{tabular}

\section{Prächolestatische PBC}

An unserer Ambulanz wurden $23 \mathrm{~Pa}$ tienten mit einer „prächolestatischen“ $\mathrm{PBC}$ über einen medianen Zeitraum von 7 Jahren (1-24 Jahre) betreut. Diese Patienten wurden getrennt ausgewertet und nicht in die Datenanalyse der cholestatischen PBC-Patienten mit einbezogen. Von diesen 23 Patienten wurden alle mit Ursodesoxycholsäure behandelt. Zwei entwickelten minimal erhöhte APWerte (112 U/l und $110 \mathrm{U} / \mathrm{l})$. Eine 3 . Patientin hatte bereits zum Zeitpunkt der Erstdiagnose bei normaler AP eine histologisch verifizierte Leberzirrhose. Diese konnte aber auf einen Overlap zur Autoimmunhepatitis zurückgeführt werden.

\section{Diskussion}

Es gibt zahlreiche Studien, die demographische Daten, Präsentation und Verlauf der PBC untersuchen. Die meisten dieser Arbeiten inkludierten aber keine Fälle aus Mitteleuropa.

Im Wesentlichen sind demographische Daten aus unserer Studie, wie das Alter bei der Erstdiagnose, Anzahl der symptomatischen Fälle und auch das Vorhandensein von AMA, mit der bisher publizierten rezenteren Literatur vergleichbar [7-9]. Wir beobachteten das Vorhandensein einer Leberzirrhose in $11 \%$, wobei die Zirrhose bei 2 Patientinnen bereits bei der Erstdiagnose bestand. Nur 2 Patientinnen (2\%) verstarben im Beobachtungszeitraum an der Erkrankung. Diese Zahlen sind deutlich niedriger als in anderen Publikationen. Die Analyse einer 770 Patienten umfassenden Kohorte aus Großbritannien ergab eine leberbe- zogene Mortalität von etwa $20 \%$ über einen medianen Beobachtungszeitraum von etwa 7 Jahren [7]. In dieser Studie hatten 5 Jahre nach Diagnosestellung bereits $12 \%$ Aszites, $7 \%$ eine hepatische Enzephalopathie, 9\% Ikterus und $15 \%$ ein Leberversagen. Unsere Ergebnisse weisen im Vergleich zu dieser britischen Arbeit auf einen deutlich milderen Verlauf hin. Dies könnte durch regionale Unterscheide in der Progression der Erkrankung erklärt werden. Limitierender Faktor unserer Arbeit hinsichtlich der Vergleichbarkeit mit den großen Kohortenstudien ist letztlich die geringe Patientenzahl mit nur 2 Todesfällen. Auch in unserer Kohorte hatten knapp 30\% der mittels FibroScan ${ }^{\circledR}$ untersuchten Patienten einen Steifigkeitswert, der mit einem deutlich erhöhten Risiko für Komplikationen assoziiert ist. Möglicherweise hätte ein längeres Follow-up die Rate an Komplikationen gerade bei diesen Patienten erhöht. Die aber in der britischen Studie publizierten hohen Komplikationsraten bereits nach 5 Jahren waren an unserem Kollektiv nicht zu beobachten.

Alle Patienten wurden mit Ursodesoxycholsäure behandelt. Auffällig war die unzureichende und nicht den Leitlinien entsprechende Dosierung bei mehr als einem Drittel der Fälle [6]. Etwa 30\% der Patienten hatten ein inadäquates biochemisches Ansprechen in Anlehnung an die Paris-II-Kriterien auf die Therapie mit Ursodesoxycholsäure. Diese Rate entspricht bisher publizierten Studien $[6,10]$. Ein direkter Vergleich der Ansprechraten in unserer Kohorte mit anderen Studien ist nicht zulässig, da sich alle publizierten Kriterien auf laborbiochemische Veränderungen 6-24 Monate nach Beginn der Ursodesoxycholsäuretherapie beziehen und diese Labordaten an diesen Zeitpunkten von uns nicht erhoben wurden. Wir fanden keinen $\mathrm{Zu}$ sammenhang zwischen der Dosis von Ursodesoxycholsäure und dem Ansprechen auf die Therapie. Nur einer von 29 Nonrespondern entwickelte eine Leberzirrhose.

Die AP-Spiegel bei Erstdiagnose und auch im Follow-up mit vorwiegender Erhöhung im Bereich des 1- bis 2-Fachen des oberen Grenzwerts des Normbereichs waren in unserer Kohorte niedriger verglichen mit älteren 
Hier steht eine Anzeige.

\section{曾 Springer}


Studien, in denen eine mediane APErhöhung vor Beginn der Therapie mit Ursodesoxycholsäure im Bereich des 4- bis 5-Fachen des oberen Grenzwerts des Normbereichs beschrieben wurde $[11,12]$. Auch in einer rezenten Metaanalyse hatten $52 \%$ aller Patienten eine AP über dem 2-Fachen und $22 \%$ über dem 4-Fachen des oberen Grenzwerts des Normbereichs [8]. Die Höhe der AP bei Diagnose und im Verlauf kann das Outcome (Lebertransplantation oder Tod) voraussagen [8]. Möglicherweise erklären die gering erhöhten AP-Werte in unseren Fällen bei der Erstdiagnose die doch deutlich bessere Gesamtprognose. Auch in unseren Fällen war eine hohe AP bei der Erstdiagnose (über dem 4-Fachen der Norm) mit dem Auftreten einer Leberzirrhose assoziiert.

AMA-Positivität ohne erhöhte Leberwerte ist nicht ausreichend, um eine PBC $\mathrm{zu}$ diagnostizieren. Nur einer von $6 \mathrm{~Pa}-$ tienten entwickelt im Lauf von 5 Jahren eine PBC [13]. Diese Rate ist in etwa mit unseren Ergebnissen in 23 Fällen prächolestatischer $\mathrm{PBC}$ vergleichbar. Einschränkend muss angeführt werden, dass alle unsere Patienten mit Ursodesoxycholsäure behandelt wurden und dass ein häufigerer Übergang in eine PBC ohne diese Therapie prinzipiell denkbar wäre.

Zusammenfassend decken sich demographische Daten und auch das Ansprechen auf die Therapie mit Ursodesoxycholsäure unserer gut charakterisierten Kohorte mit den bisher publizierten Studien. Wesentliche Unterschiede zur Literatur sind eine deutlich niedrigere AP bei der Erstdiagnose und - wahrscheinlich auch dadurch bedingt - ein besseres Outcome. Bei einer Erhöhung der AP über dem 4-Fachen der Norm bei Erstdiagnose war bei unseren Fällen das Auftreten einer Leberzirrhose deutlich wahrscheinlicher, während ein inadäquates Ansprechen auf Ursodesoxycholsäure nicht mit vermehrtem Auftreten einer Zirrhose assoziiert war. Soweit von den Grazer Daten auf Österreich geschlossen werden kann, scheint die PBC hierzulande milder $\mathrm{zu}$ verlaufen als in anderen geographischen Regionen.

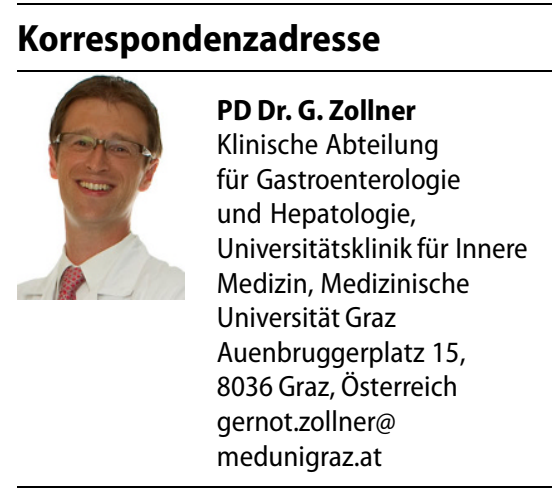

Funding. Open access funding provided by Medical University of Graz.

\section{Einhaltung ethischer Richtlinien}

Interessenkonflikt. G. Zollner, J. Prattes und A. Nemeth geben an, dass kein Interessenkonflikt besteht.

Bei diesem Beitrag handelt es sich um eine retrospektive Studie, die von der zuständigen Ethikkommission genehmigt wurde (Votum: 26-245 ex 13/14).

Open Access Dieser Artikel wird unter der Creative Commons Namensnennung 4.0 International Lizenz (http://creativecommons.org/licenses/by/4.0/deed. de) veröffentlicht, welche die Nutzung, Vervielfältigung, Bearbeitung, Verbreitung und Wiedergabe in jeglichem Medium und Format erlaubt, sofern Sie den/die ursprünglichen Autor(en) und die Quelle ordnungsgemäßnennen, einen Link zur Creative Commons Lizenz beifügen und angeben, ob Änderungen vorgenommen wurden.

\section{Literatur}

1. Kaplan MM, Gershwin ME (2005) Primary biliary cirrhosis. NEngl J Med 353:1261-1273

2. Beuers $U$, Gershwin ME, Gish RG, Invernizzi $P$, Jones DE, Lindor K, Ma X et al (2015) Changing nomenclature for PBC: From "cirrhosis" to "cholangitis". Hepatology 62:1620-1622

3. EASL Clinical Practice Guidelines (2009) Management of cholestatic liver diseases. J Hepatol 51:237-267

4. Ludwig J, Dickson ER, McDonald GS (1978) Staging of chronic nonsuppurative destructive cholangitis (syndrome of primary biliary cirrhosis). Virchows Arch APathol Anat Histol 379:103-112

5. Corpechot C, Carrat F, Poujol-Robert A, Gaouar F, Wendum D, Chazouilleres O, Poupon R (2012) Noninvasive elastography-based assessment of liver fibrosis progression and prognosis in primary biliary cirrhosis. Hepatology 56:198-208

6. European Association for the Study of the Liver (2017) Electronic address eee, European Association for the Study of the L. EASL Clinical Practice Guidelines: The diagnosis and management of patients with primary biliary cholangitis. J Hepatol 67:145-172

7. Prince $M$, Chetwynd A, Newman W, Metcalf JV, James OF (2002) Survival and symptom progression in a geographically based cohort of patients with primary biliarycirrhosis: follow-upfor up to 28 years. Gastroenterology 123:1044-1051

8. Lammers WJ, van Buuren HR, Hirschfield GM Janssen $\mathrm{HL}$, Invernizzi P, Mason AL, Ponsioen $\mathrm{CY}$ et al (2014) Levels of alkaline phosphatase and bilirubin are surrogate end points of outcomes of patients with primary biliary cirrhosis: an international follow-up study. Gastroenterology 147:1338-1349e5 (quiz e1315)

9. Lleo A, Marzorati S, Anaya JM, Gershwin ME (2017) Primary biliary cholangitis: a comprehensive overview. Hepatol Int 11:485-499

10. Carbone M, Mells GF, Pells G, Dawwas MF, Newton $\mathrm{JL}$, Heneghan MA, Neuberger JM et al (2013) Sex and age are determinants of the clinical phenotype of primary biliary cirrhosis and response to ursodeoxycholic acid. Gastroenterology 144(e567):560-569e7 (quize513-564)

11. Poupon RE, Lindor KD, Cauch-DudekK, Dickson ER, Poupon R, Heathcote EJ (1997) Combined analysis of randomized controlled trials of ursodeoxycholic acid in primary biliary cirrhosis. Gastroenterology 113:884-890

12. Poupon RE, Lindor KD, Pares A, Chazouilleres $O$, Poupon R, Heathcote EJ (2003) Combined analysis of the effect of treatment with ursodeoxycholic acid on histologic progression in primary biliary cirrhosis. JHepatol 39:12-16

13. Dahlqvist G, Gaouar F, Carrat F, Meurisse $S$, Chazouilleres O, Poupon R, Johanet $C$ et al (2017) Large-scale characterization study of patients with antimitochondrial antibodies but nonestablished primary biliary cholangitis. Hepatology 65:152-163 